\title{
Imaging and photometry of nearby dwarf galaxies
}

\section{Southern dwarfs ${ }^{\star}, \star \star$}

\author{
L. N. Makarova ${ }^{1,2}$, I. D. Karachentsev ${ }^{1}$, E. K. Grebel ${ }^{3}$, D. Harbeck ${ }^{4}$, G. G. Korotkova ${ }^{1}$, and D. Geisler ${ }^{5}$ \\ 1 Special Astrophysical Observatory of the Russian Academy of Sciences, Nizhnij Arkhyz 369167, Karachaevo-Cherkessia, \\ Russia \\ e-mail: lidia@sao.ru; ikar@luna.sao.ru \\ 2 Isaac Newton Institute of Chile, SAO Branch \\ 3 Astronomical Institute, University of Basel, Venusstrasse 7, 4102 Binningen, Switzerland \\ e-mail: grebel@astro.unibas.ch \\ ${ }^{4}$ Department of Astronomy, University of Wisconsin, 475 North Charter Street, Madison, WI 53706-1582, USA \\ e-mail: harbeck@astro.wisc.edu \\ 5 Departamento de Física, Grupo de Astronomía, Universidad de Concepción, Casilla 160-C, Concepción, Chile \\ e-mail: doug@kukita.cfm.udec.cl
}

Received 13 February 2004 / Accepted 6 December 2004

\begin{abstract}
We carried out CCD photometry in the Johnson-Cousins $B$ and $R$ bands of 23 dwarf galaxies: SDIG, ESO 410-17, KK11, ESO 245-05, KKs3, KK27, KK38, KK40, IC 4662, KK244, KK246, KK247, KK248, KK249, KK253, KK255, KK256, KK257, KK258, KK259, UGCA 438, ESO 347-17, and UGCA 442. Both isolated galaxies and members of the Sculptor group and the NGC 1313 group were observed. The galaxy sample is characterized by a median distance of $9.3 \mathrm{Mpc}$, and median absolute magnitude of $-14.8 \mathrm{mag}$. The central surface brightnesses are in the range from 22.2 to $24.4 \mathrm{mag} \operatorname{arcsec}^{-2}$ in $B$.
\end{abstract}

Key words. galaxies: dwarf - galaxies: photometry - galaxies: irregular - galaxies: general - galaxies: evolution

\section{Introduction}

The Local Volume $(\leq 10 \mathrm{Mpc})$ is an ideal laboratory for the investigation of structure, kinematics and dynamics of galaxies and their parent groups. In particular, it allows us to study objects of extremely low luminosity and surface brightness, which is the most numerous class of galaxies but which become inaccessible for detailed study when at large distances. The study of the nearby universe also allows us to probe the largescale structure and galaxy properties at the present epoch and thus serves as a reference for evolutionary studies. Recent systematic surveys for dwarf galaxies roughly doubled the number of objects known in the Local Volume (see Karachentseva \& Karachentsev 1998, 2000; Karachentsev et al. 2000a; Whiting et al. 2002; Karachentsev et al. 2004). Many of the new dwarf galaxies are situated within the boundaries of the known nearby groups. Measurements of accurate distances and radial

\footnotetext{
* Based on observations obtained with CTIO 1.5-m telescope, which is operated by the Association of Universities for Research in Astronomy Inc. (AURA), under a cooperative agreement with the National Science Foundation as part of the National Optical Astronomy Observatories.

$\star \star$ Tables 1 and 2, complete Figs. 1 and 2 are only available in electronic form at http://www. edpsciences .org
}

velocities of these galaxies are essential to understand the structure and dynamics of nearby galaxy groups.

Enormous progress has been made recently in accurate distance measurements for nearby galaxies beyond the Local Group (LG) based on the luminosity of the tip of the red giant branch (TRGB). Over the last four years, our snapshot surveys of nearby galaxies using WFPC2 aboard the Hubble Space Telescope (HST) have provided us with distances for a hundred nearby galaxies obtained with an accuracy of about $10 \%$ based on the TRGB method (Karachentsev et al. 2000b, 2002a,b,c, 2003a,b,c,d). Further significant progress is expected in the near future due to observations with the Advanced Camera for Surveys (ACS) aboard the HST.

Hopp \& Schulte-Ladbeck (1995), Karachentseva et al. (1996), Bremnes et al. (1998, 1999), Makarova (1999), Jerjen et al. (2000), Barazza et al. (2001), Makarova et al. (2002) and Parodi et al. (2002) presented results of CCD surface photometry of many dwarf galaxies within and outside the nearby groups with distances $\leq 10 \mathrm{Mpc}$. Despite this considerable observational progress, for more than $3 / 4$ of the dwarf galaxies in the Local Volume neither surface brightness profiles nor magnitudes and colors have been measured yet, nor have these galaxies been imaged with modern CCD detectors or reliably classified by morphological type. We are therefore 

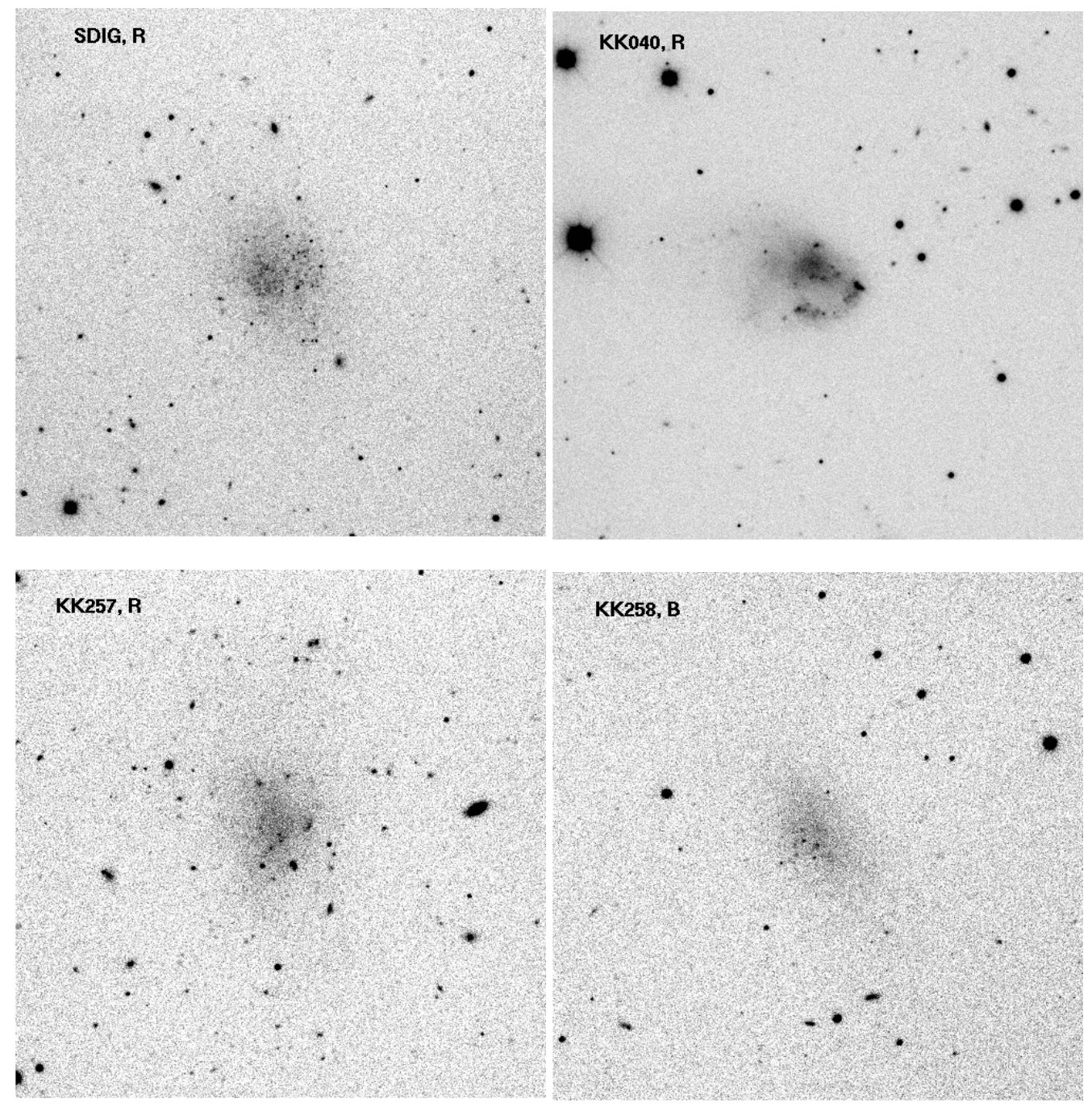

Fig. 1. The images of the 4 sample galaxies. The field size is $4^{\prime} \times 4^{\prime}$, North is up and East is left.

carrying out follow-up observations of nearby dwarf galaxies using medium-size ground based telescopes (see Grebel et al. 2000a; Lee et al. 2003).

This is the second paper in a series of articles, intended to present results from the follow-up observations of new nearby galaxies and to obtain their general photometric parameters. In the first paper (Makarova et al. 2002) we described photometry of 16 new nearby galaxies observed at the 3.5-m telescope of Apache Point Observatory. Here we present photometric data for 23 more dwarf galaxies.

\section{Observations and data reduction}

The galaxies were selected from the lists of Karachentseva \& Karachentsev $(1998,2000)$. Some nearby dwarf objects from the ESO catalog (Lauberts 1982) were added to the observational program. These objects were observed using the $1.5-\mathrm{m}$ telescope at the Cerro Tololo Inter-American Observatory (CTIO) in Chile from 30 August 2000 to 1 September 2000. We carried out direct imaging of the galaxies using the Cassegrain focus CCD Imager (CFCCD), which is equipped with a thinned, anti-reflection coated SITe CCD $(2048 \times 2048$ pixels, pixel scale $0.24^{\prime \prime}$ pixel $^{-1}$, field of view $8^{\prime} \times 8^{\prime}$ ). The observing $\log$ is presented in Table ??. Our data were obtained using Johnson $B$ and Kron-Cousins $R$ filters. The seeing of the frames is indicated in Table ??. These values were measured in the resulting bias-subtracted, flat-fielded and co-added images. Bias frames and twilight flats were obtained each night. Also, several Landolt (1992) standard fields were observed each night covering a range of airmasses. To transform our instrumental magnitudes to the standard Johnson-Cousins system, zero points, color coefficients and extinction coefficients were determined from the standard stars observed in the third, clear photometric night. The uncertainties of the transformation to the standard system are 0.05 in the $B$ filter and 0.03 in the $R$ filter. Subsequently, by consulting the CTIO web pages (http://www.ctio.noao.edu/site/phot/ sky_conditions.html), we discovered that during the the first night, $30 \%$ to $40 \%$ of the sky was covered by clouds. On the second night, the cloud cover ranged from zero to $40 \%$. Hence while data were obtained during these two nights, their photometric quality is questionable. Nonetheless, we attempted a photometric transformation. We used the extinction coefficients and the color terms from the third night, and zero 
points obtained from the appropriate standard field closest to the time of the respective observations. Our measurements show that the objects from the second night have a quite good calibration. The mean obtained zero point in $B$ is equal to 23.14 with a scatter of 0.05 , which agrees well with the zero point of 23.19 obtained in the photometric night. Therefore, photometric conditions look quite stable and good during this night. The one exception is the standard field exposured in the evening soon after twilight flats. The zero points obtained from this field show additional extinction of about 0.39 mag in $B$. Total magnitudes, colors and central surface brightness of galaxies exposured near this time of the night are marked with a colon in Table ??. The data from the first night may be seriously affected by clouds. However, the standard field exposured in the middle of the night does not show additional atmospheric extinction with the measured zero point in $B$ equal to 23.15. The zero points obtained in the last part of this last night are affected by clouds with a possible additional extinction of $0.33 \mathrm{mag}$ in $B$. The data obtained from the images of this night are marked with a colon in Table ??, except for ESO 347-G017 and ESO 410-G017, which were apparently obtained under good photometric conditions.

The images were processed using the MIDAS package developed at the European Southern Observatory (ESO). After bias subtraction and flat-fielding, we removed cosmic ray hits, and then images obtained in the same filter were co-added. The resulting images of the sample galaxies are presented in Fig. 1; the full set of 23 galaxies is available electronically. Background stars were removed from the frames and the sky background on the image was approximated by a tilted plane. The data reduction procedure and its characteristic uncertainties (which are similar in both studies) were described in detail in the first article of the series (Makarova et al. 2002).

\section{Photometry}

To measure total galaxy magnitudes in each band, integrated photometry was performed in circular apertures with increasing radii from a pre-chosen center to the faint outskirts of the galaxies. The total magnitude was then estimated as the asymptotic value of the obtained radial growth curve. The internal uncertainties of the total magnitude determination were 0.05 in $B$ and 0.08 in $R$. The measurement results are summarized in Table ??. There we list:

(1) galaxy name;

(2), (3) equatorial coordinates for the epoch J2000;

(4) morphological type in de Vaucouleurs' notation;

(5) radial velocity (in $\mathrm{km} \mathrm{s}^{-1}$ ) with respect to the Local Group centroid with the apex parameters from Karachentsev \& Makarov (1996). The source of the radial velocity is the NASA Extragalactic Database (NED) or other recent sources, as noted in the table;

(6) distance to the galaxy in Mpc with indication of the method used: "rgb" - from the luminosity of the tip of red giant branch, "tf" - from the Tully-Fisher relation, "mem" - from membership in the known groups; "h"-from the Hubble relation with the Hubble parameter $H_{0}=72 \mathrm{~km} \mathrm{~s}^{-1} \mathrm{Mpc}^{-1}$;

(7) Galactic extinction in the $B$-band from Schlegel et al. (1998);

(8) integrated apparent magnitude in the $B$-band from the NASA Extragalactic Database (NED). The sources of the photometric data are noted, where this is possible;

(9) integrated $B$-magnitude from our measurements;

(10) integrated $(B-R)$ color from our photometry;

(11) central surface brightness in the $B$-band in mag $\operatorname{arcsec}^{-2}$. The surface brightness values were estimated by eye from the surface brightness profiles;

(12) Holmberg diameter in arcmin;

(13) the galaxy's absolute magnitude.

Seven galaxies from our sample have a large $B$-magnitude difference $(\geq 0.5 \mathrm{mag}$ ) from the NED values. The sources for three of them (E410-17, KK255 and KK258) are not indicated in NED. The total $B$ magnitude value in NED is 19.8 for KK257. It comes from digitized photographic plates and seems too faint. The remaining three galaxies (KK246, KK249 and KK253) have NED values taken from the UGC catalog. Except for KK255, these galaxies were observed under good photometric conditions. We conclude based on this that our results are the most reliable and adopt them in our subsequent discussion.

Azimuthally averaged surface brightness profiles for our galaxies were obtained by differentiating the galaxy growth curves with respect to radius (Bremnes et al. 1998; Makarova 1999). The resulting profiles in the $B$ and $R$ colors are displayed in Fig. 2. Most of the galaxies were measured to a level of 26 to $28 \mathrm{mag} \operatorname{arcsec}^{-2}$ in the $B$ filter. Mean uncertainties of the measurements were estimated by intercomparison of individual profiles for the same objects obtained from different frames in the same passband. They amount to about 0.04 at the $23 \mathrm{mag} \mathrm{arcsec}^{-2}$ isophotal level and about 0.3 to 0.5 at the $27 \mathrm{mag} \operatorname{arcsec}^{-2}$ isophotal level in each of the filters. The illustrative error bars are also shown in Fig. 2.

By summing up the intrinsic errors indicated above, we derive the final uncertainties of estimating total magnitudes: 0.17 in the $B$ filter and 0.19 in $R$. The final uncertainties in deriving surface brightness depend on the surface brightness level of the galaxy itself. The typical errors at the level of $23 \mathrm{mag} \mathrm{arcsec}^{-2}$ are 0.16 in the $B$ filter and 0.18 in $R$. They rise to about 0.19 in $B$ and 0.22 in $R$ at the level of 25-26 mag $\operatorname{arcsec}^{-2}$ and amount to about 0.40 to 0.50 in $B$ at the level of 27-28 mag $\operatorname{arcsec}^{-2}$. The uncertainties in the $R$ filter are larger, and reach about $0 .{ }^{\mathrm{m}} 6$ at the level of 27-28 mag $\operatorname{arcsec}^{-2}$.

\section{Notes on individual galaxies}

Below, some properties of the galaxies are briefly discussed.

SDIG = Sculptor dwarf irregular galaxy = ESO 349031. A dwarf companion to the bright spiral galaxy NGC 7793 in the Sculptor group. A sprinkling of luminous stars is seen in the main body of this dIrr. Its distance was estimated from the Tully-Fisher relation by Karachentsev et al. (2003a). Surface photometry of the galaxy was performed by 

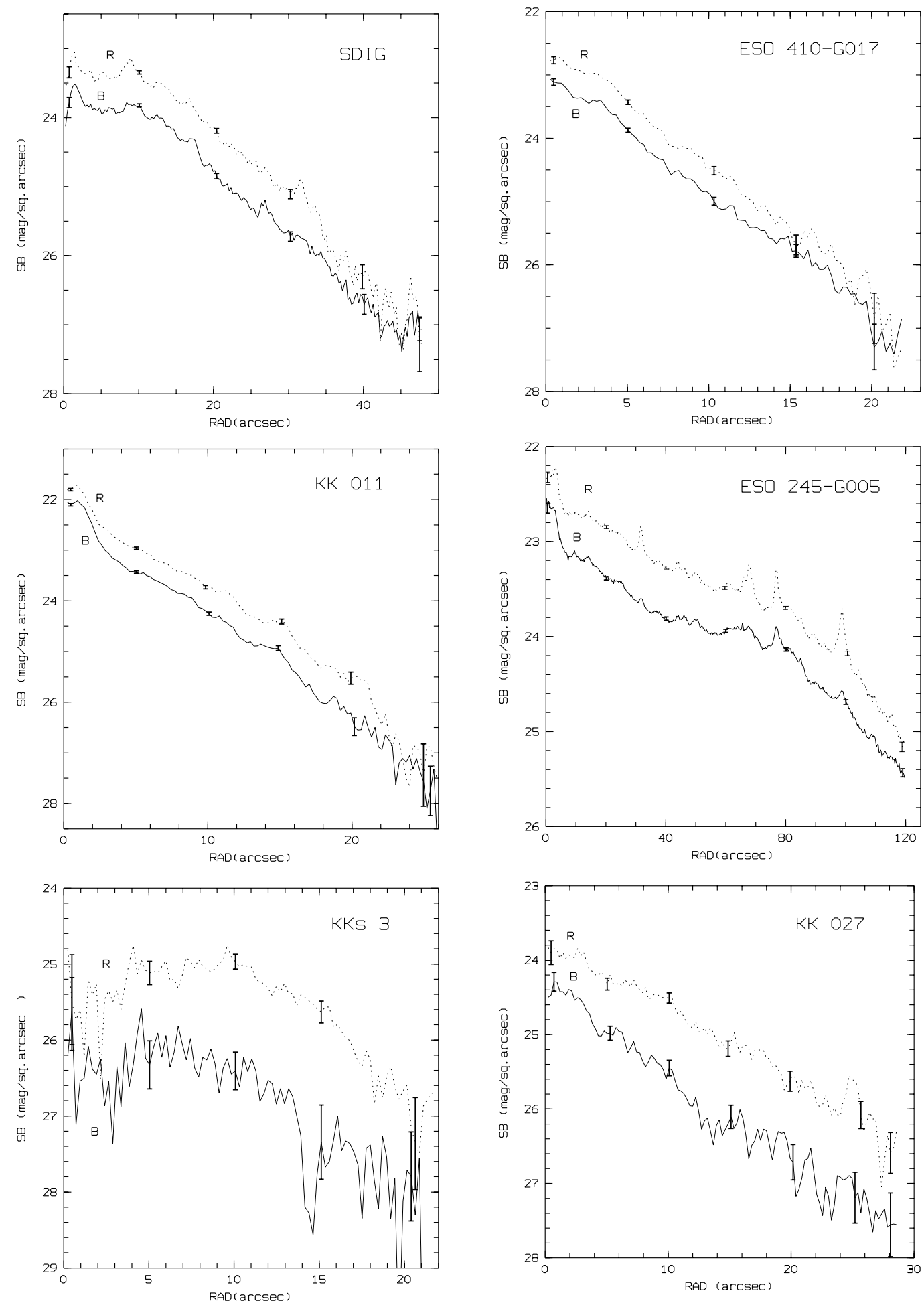

Fig. 2. The azimuthally averaged surface brightness profiles for the nearby dwarf galaxies.

Heisler et al. (1997). The comparison of their total $B$ magnitude and central surface brightness with our measurements yields a relatively good agreement: $B_{\text {Heisler }}-B_{\text {our }}=-0.14$ and $\mu(0)_{\text {Heisler }}^{B}-\mu(0)_{\text {our }}^{B}=-0.1$. However, the agreement of the total colors is worse $(B-R)_{\text {Heisler }}-(B-R)_{\text {our }}=0.26$.

ESO 245-05. A dwarf companion to NGC 625 in the Sculptor group. Its TRGB distance was measured by
Karachentsev et al. (2003a). The luminous central region may be an HII region or a nucleus.

KKs3. A galaxy of extremely low surface brightness with a star and a 2 MASS source superposed on its central part. An unreliable distance estimate, $4.1 \mathrm{Mpc}$, is based on a probable membership of the object in the NGC 1313 group.

KK 027. A companion to NGC 1313. It was classified by Karachentsev et al. (2003b) as dSph, however, its 
color-magnitude diagram (obtained with the WFPC2 aboard HST) contains a small number of bluish stars. There is faint HI emission from KK 027 in HIPASS with $V_{\mathrm{h}}=+560 \mathrm{~km}$ $\mathrm{s}^{-1}$. Like LGS3, it probably belongs to the transition type of dIrr/dSph galaxies (see Grebel et al. 2003 for a more extensive discussion of these dwarfs).

IC 4662. A bright irregular galaxy with a prominent complex of blue stars on the northern side. This galaxy, resolved into stars, has no distance estimate, in spite of its very low radial velocity.

KK 246 = ESO 461-036. An irregular dwarf galaxy situated at the edge of the Local Void (Tully 1988). A few faint diffuse knots are visible.

KK 253 = ESO 074-02. Faint HI emission with $V_{\mathrm{h}}=3140$, $W_{50}=50 \mathrm{~km} \mathrm{~s}^{-1}$ is seen in HIPASS. The optical image shows a few faint, small, diffuse structures in this galaxy.

KK 255 = ESO 146-02. There is a faint very diffuse curved tail towards the NW. The magnitude of 14.6 mag in the NED seems too luminous as compared to our measurements.

KK 257 = ESO 238-05. The magnitude of 19.83 in the NED is apparently wrong.

KK 258 = ESO 468-020. A peripheral member of the Sculptor group, semi-resolved into stars. It looks like a fairly nearby dwarf galaxy, based on its angular extent and degree of resolution. It was not detected in HIPASS.

KK $259=$ ESO 291-07. $V_{\mathrm{h}}=1799 \pm 899 \mathrm{~km} \mathrm{~s}^{-1}$ in the NED. According to HIPASS it has $V_{\mathrm{h}}=1578 \mathrm{~km} \mathrm{~s}^{-1}$ and $W_{50}=47 \mathrm{~km} \mathrm{~s}^{-1}$. This galaxy has a prominent bar and several luminous regions that look like active HII regions.

UGCA 438. The object is situated in front of the Sculptor group (Karachentsev et al. 2002c) and is clearly resolved into stars. Surface and integrated photometry of the galaxy was performed by Lee and Byun (1999). The authors note that it was very difficult to reliably derive the surface photometry of UGCA 438 because of the presence of a very bright foreground star close to the center of the galaxy. Nevertheless, the comparison of their total $B$ magnitude and color with our measurements yields a relatively good agreement: $B_{\mathrm{Lee}}-B_{\text {our }}=-0.18$ and $(B-R)_{\text {Lee }}-(B-R)_{\text {our }}=-0.14$.

UGCA $442=$ RFGC 4177. This flat edge-on galaxy is a companion to NGC 7793 (Karachentsev et al. 2003a).

\section{Discussion}

In this work we present imaging and general photometric parameters of 23 southern dwarf galaxies, most of them only recently found to be relatively nearby objects $(\leq 10 \mathrm{Mpc})$. Both isolated galaxies as well as members of the Sculptor group and of the NGC 1313 group were observed. The azimuthally averaged surface brightness profiles in $B$ and $R$ generally exhibit similar shapes and slopes. Dwarf irregular galaxies with bars and/or prominent star formation show larger differences in their profiles in these two wavebands. About half of the galaxies under investigation demonstrate a minor increase in the redness of the total color index $B-R$ from the center to the periphery. Here we take into account only the cases where the color gradient can be easily distinguished from the inner parts of the galaxies, and not any trend for the most outer part of the profiles only, where errors of the night sky play a significant role in the color gradients. This gradient is likely to be a result of the increase in the average age of the stellar population towards the edge of the galaxy (Makarova 1999; Makarova et al. 2002). In particular, a more extended old stellar population is a common property of dwarf galaxies in the Local Group (Grebel 1997, 2000b). The absence of a noted color gradient for half of the galaxies of our sample may be indicative of a more homogeneous spatial distribution of the stellar populations of different ages in these dwarf galaxies, or of a small age and metallicity spread.

The profiles of one of the dSph galaxies, KK 027, illustrate the concentration of younger, bluish stars toward the center of this dwarf as found also from resolved color-magnitude diagrams (Karachentsev et al. 2003b), indicative of the population gradients found in a number of nearby dSphs (Harbeck et al. 2001). No such trend is apparent for the dSphs KK 244 and KK 258.

As can be seen from Fig. 2, most of the surface brightness profiles have a linear outer part, which can be considered as evidence for the presence of an underlying stellar disc component of the galaxy. About $70 \%$ of the surface brightness profiles show central light depression, which is very common for dwarf irregular galaxies. We also have three profiles in our samples that seem purely exponential, and three of the galaxies have prominent light excesses in their central parts.

Here, we give some general comments concerning the galaxies observed. About $80 \%$ of the 23 galaxies studied are irregular objects of low surface brightness and in many of them the brightest stars are resolved. The galaxy central surface brightnesses are mostly distributed in the interval from 22.2 to $24.4 \mathrm{mag} \mathrm{arcsec}^{-2}$ in $B$. Two galaxies of the sample have a central surface brightness out of this range: IC 4662 (20.7) and the dSph KKs 3 (26.0). The latter value is unusually faint even for a dSph (e.g., Grebel et al. 2003; Zucker et al. 2004) - on the other hand, these data were obtained during the first night and may have been affected by clouds. The absolute $B$ magnitudes occupy a rather wide range from about $-9^{\mathrm{m}} .8$ to $-17^{\mathrm{m}} \mathrm{.}$, similar to the range of absolute magnitudes found among Local Group dSphs and dIrrs (see Grebel et al. 2003). The galaxy sample has a median distance of $9.3 \mathrm{Mpc}$, and a median absolute magnitude of $-14.8 \mathrm{mag}$, which reflects the fact that our sample consists primarily of dIrrs.

Acknowledgements. This research has made use of the NASA/IPAC Extragalactic Database (NED) which is operated by the Jet Propulsion Laboratory, California Institute of Technology, under contract with the National Aeronautics and Space Administration. In this work we use data of HIPASS, which is operated by the Australia Telescope National Facility, CSIRO. The work was supported by RFBR grant 01-16001. EKG acknowledges partial support from the Swiss National Science Foundation. D.G. gratefully acknowledges support from the Chilean Centro de Astrofísica FONDAP No. 15010003.

\section{References}

Barazza, F. D., Binggeli, B., \& Prugniel, P. 2001, A\&A, 373, 12 Bremnes, T., Binggeli, B., \& Prugniel, P. 1998, A\&AS, 129, 313 
Bremnes, T., Binggeli, B., \& Prugniel, P. 1999, A\&AS, 137, 337

Cote, S., Freeman, K. C., Carignan, C., \& Quinn, P. J. 1997, AJ, 114, 1313

da Costa, L. N., Pellegrini, P. S., Davis, M., et al. 1991, ApJS, 75, 935

Fouqué, P., Durand, N., Bottinelli, L., Gouguenheim, L., \& Paturel, G. 1992, Catalogue of Optical Radial Velocities, Observatories de Lyon et Paris-Meudon, 1, 1

Grebel, E. K. 1997, Rev. Mod. Astron., 10, 27

Grebel, E. K., Seitzer, P., Dolphin, A. E., et al. 2000a, in Stars, Gas and Dust in Galaxies: Exploring the Links, ed. D. Alloin, K. Olsen, \& C. Galaz (San Francisco: ASP), ASP Conf. Ser., 221147

Grebel, E. K. 2000b, in 33rd ESLAB Symp., Star formation from the small to the large scale, ed. F. Favata, A. A. Kaas \& A. Wilson (Noordwijk: ESA), ESA SP-445, 87

Grebel, E. K., Gallagher, J. S., \& Harbeck, D. 2003, AJ, 125, 1926

Harbeck, D., Grebel, E. K., Holtzman, J., et al. 2001, AJ, 122, 3092

Heisler, C. A., Hill, T. L., McCall, M. L., \& Hunstead, R. W. 1997, MNRAS, 285, 374

Hopp, U., \& Schulte-Ladbeck, R. E. 1995, A\&AS, 111, 527

Huchtmeier, W. K., Karachentsev, I. D., \& Karachentseva, V. E. 2003, A\&A, 401, 483

Huchtmeier, W. K., Karachentsev, I. D., \& Karachentseva, V. E. 2001, A\&A, 377, 801

Huchtmeier, W. K., Karachentsev, I. D., Karachentseva, V. E., \& Ehle, M. 2000, A\&AS, 141, 469

Jerjen, H., Binggeli, B., \& Freeman, K. C. 2000, AJ, 119, 593

Karachentseva, V., Prugniel, P., Vennik, J., et al. 1996, A\&AS, 117, 343

Karachentseva, V. E., \& Karachentsev, I. D. 1998, A\&AS, 127, 409

Karachentseva, V. E., \& Karachentsev, I. D. 2000, A\&AS, 146, 359

Karachentsev, I. D., \& Makarov, D. I. 1996, AstL, 22, 455

Karachentsev, I. D., \& Karachentseva, V. E. 1999, A\&A, 341, 355

Karachentsev, I. D., Karachentseva, V. E., Suchkov, A. A., \& Grebel, E. K. 2000a, A\&AS, 145, 415

Karachentsev, I. D., Karachentseva, V. E., Dolphin, A. E., et al. 2000b, A\&A, 363, 117

Karachentsev, I. D., Dolphin, A. E., Geisler, D., et al. 2002a, A\&A, 383,125
Karachentsev, I. D., Sharina, M. E., Dolphin, A. E., et al. 2002b, A\&A, 385,21

Karachentsev, I. D., Sharina, M. E., Makarov, D. I., et al. 2002c, A\&A, 389,812

Karachentsev, I. D., Grebel, E. K., Sharina, M. E., et al. 2003a, A\&A, 404, 93

Karachentsev, I. D., Makarov, D. I., Sharina, M. E., et al. 2003b, A\&A, 398,479

Karachentsev, I. D., Sharina, M. E., Dolphin, A. E., \& Grebel, E. K. 2003c, A\&A, 408, 111

Karachentsev, I. D., Sharina, M. E., Dolphin, A. E., et al. 2003d, A\&A, 398, 467

Karachentsev, I. D., Karachentseva, V. E., Huchtmeier, W. K., \& Makarov, D. I. 2004, AJ, accepted

Landolt, A. 1992, AJ, 104, 340

Lauberts, A. 1982, ESO/Uppsala survey of the ESO(B) atlas, Garching: European Southern Observatory (ESO)

Lauberts, A., \& Valentijn, E. A. 1989, The surface photometry catalogue of the ESO-Uppsala galaxies, Garching bei Munchen: European Southern Observatory

Lee, M. G., \& Byun, Y.-I. 1999, AJ, 118, 817

Lee, H., Grebel, E. K., \& Hodge, P. W. 2003, A\&A, 401, 141

Makarov, D. I., Karachentsev, I. D., \& Burenkov, A. N. 2003, A\&A, 405, 951

Maddox, S. J., Sutherland, W. J., Efstathiou, G., \& Loveday, J. 1990, MNRAS, 243, 692

Makarova, L. 1999, A\&AS, 139, 491

Makarova, L., Karachentsev, I., Grebel, E., \& Barsunova, O. 2002, A\&A, 384, 72

Parodi, B. R., Barazza, F. D., \& Binggeli, B. 2002, A\&A, 388, 29

Schlegel, D. J., Finkbeiner, D. P., \& Davis, M. 1998, ApJ, 500, 525

Tully, R. B. 1988, Nearby Galaxy Catalog (Cambridge Univ. Press)

De Vaucouleurs, G., De Vaucouleurs, A., Corwin Jr., H.G., et al. 1991, Third Reference Catalogue of Bright Galaxies, version 3.9

Whiting, A. B., Hau, G. K. T., \& Irwin, M. 2002, ApJS, 141, 123

Zucker, D. B., Kniazev, V., Bell, E. F., et al. 2004, ApJ, 612, L121 